\title{
ON THE MALCEV COMPLETION OF KÄHLER GROUPS
}

\author{
JAUme Amorós \\ Universitat de Barcelona \\ Dep. d'Algebra i Geometria - Fac. de Matemàtiques \\ Gran Via 585 - 08007 Barcelona, Spain \\ amoros@cerber.mat.ub.es
}

$22 / 6 / 95$

\section{Introduction.}

The study of compact Kähler manifolds made by Hodge and others shows that a Kähler structure imposes very strong conditions on the homotopy type of a compact complex manifold $X$. In particular, unlike in the case of compact differentiable or closed complex manifolds, not every finitely presented group $G$ is the fundamental group of a compact Kähler manifold. Such groups are called Kähler groups.

This note has been inspired by the recent work of F. Johnson and E. Rees ([JR]) and M. Gromov ([G]), showing that free products, and in particular free groups, are not Kähler. It has been our purpose to extend this result and find other restrictions on the presentations of Kähler groups. This is done by translating properties of cup products in $H^{*}(X)$ into properties of the group bracket in $\pi_{1} X$, an idea that came out of $[\mathrm{JR}]$, and also by examining the Albanese map $X \rightarrow A l b(X)$ after $[\mathrm{C}]$. We describe an algorithm derived from $[\mathrm{St}]$ to compute $\Gamma_{1} / \Gamma_{2} G, \Gamma_{2} / \Gamma_{3} G \otimes \mathbb{R}$ from a given presentation of a group $G$, and use it to give three conditions for the groups to be Kähler: The Lie algebra $\mathcal{L}_{2} G$, equivalent to the holonomy algebra, cannot be free (3.3); one- or two-relator Kähler groups either have a torsion abelianized or have a Malcev completion isomorphic to that of a compact Riemann surface group (4.7); nonfibered Kähler groups must satisfy certain lower bounds for the number of their defining relations, equivalently upper bounds for the rank of $\Gamma_{2} / \Gamma_{3} G(5.6,5.7)$.

In $\S 1$ we recall the real Malcev completion $G \otimes \mathbb{R}$ of a group $G$, its equivalent Lie algebra $\mathcal{L} G$, and a 2-step nilpotent Lie algebra $\mathcal{L}_{2} G \cong\left(\Gamma_{1} / \Gamma_{2} G \otimes \mathbb{R}\right) \oplus\left(\Gamma_{2} / \Gamma_{3} G \otimes \mathbb{R}\right)$, which is determined by $\left(\Gamma_{1} / \Gamma_{3} G\right) /$ Torsion and is equivalent to the cup products $\bigwedge^{2} H^{1}(X) \rightarrow H^{2}(X)$. This algebra is actually equivalent to the holonomy algebra of $G$ (cf. [Ch], [Ko]), and is more convenient for our computations. By [M2],[DGMS],

Supported by the Generalitat de Catalunya.

Partially supported by DGCYT grant PB93-0790

Typeset by $\mathcal{A}_{\mathcal{M}} \mathcal{S}^{\mathcal{-}} \mathrm{T}_{\mathrm{E}} \mathrm{X}$ 
when $G$ is a Kähler group the algebra $\mathcal{L}_{2} G$ determines the Malcev completion $G \otimes \mathbb{R}$. In $\oint 2$ we briefly recall Sullivan's 1 -minimal model of $X$, its duality with $\mathcal{L} \pi_{1} X$, and how the algebra $\mathcal{L}_{2} \pi_{1} X$ and the product map $\cup: \bigwedge^{2} H^{1}(X) \rightarrow H^{2}(X)$ are equivalent.

In $\S 3$ we use these results to show that if $\mathcal{L}_{2} G \cong \mathcal{L}_{2} F_{n}$, where $F_{n}$ is a finite rank free group then $G$ is not Kähler. This is a strong quantitative restriction, since the generic group presented with few relations verifies $\mathcal{L}_{2} \cong \mathcal{L}_{2} F_{n}$ for some $n$ (see Remark 1.15).

The groups with the simplest presentation after free groups are 1- and 2-relator groups. In Theorem 4.7 we determine the Malcev completions of 1- and 2-relator Kähler groups, which to a great extent characterize the groups themselves. The mean to do this is to bound from above $\operatorname{dim} \Gamma_{2} / \Gamma_{3} \pi_{1} X \otimes \mathbb{R}$ for any Kähler group $G$ by a function of the dimension of the image $Y$ of $X$ by its Albanese map $\alpha: X \rightarrow$

Alb $(X)$. A desingularization $\tilde{Y}$ of $\alpha(X)$ has been shown by F. Campana $([\mathrm{C}])$ to verify $\pi_{1} X \otimes \mathbb{R} \cong \pi_{1} \tilde{Y} \otimes \mathbb{R}$. It turns out of our work that as $\operatorname{dim} Y$ increases linearly, $\operatorname{dim} \bigwedge^{2} H^{1}(X)-\operatorname{dim} \Gamma_{2} / \Gamma_{3} \pi_{1}(X) \otimes \mathbb{R}$ grows quadratically (Prop. 4.6).

Finally, in $§ 5$, we have established a distinction between fibered and nonfibered Kähler groups, and used the mentioned techniques to give upper bounds for dim $\Gamma_{2} / \Gamma_{3} G \otimes \mathbb{R}$ for nonfibered groups, which imply lower bounds for their number of defining relations (Prop. 5.6, Cor. 5.7). Specifically, in Cor. 5.7 we give the following lower bound for the difference between the number of generators $n$ and of relations $s$ of any presentation of a nonfibered Kähler group

$$
s-n \geq 4 q-7
$$

where $q=\frac{1}{2} b_{1}(G)$. This improves a bound given by Green and Lazarsfeld ([GL]).

To proof our results and make them effective, we give in $\S 1$ an algorithm for computing $\Gamma_{1} / \Gamma_{2} G \otimes \mathbb{R}, \Gamma_{2} / \Gamma_{3} G \otimes \mathbb{R}$ and $\mathcal{L}_{2} G$ from a given presentation of $G$. This algorithm, which is easy to implement by means of the Magnus algebra of free groups, is derived from a spectral sequence given in [St], and was communicated to the author by M. Hartl. We use it in Cor. 1.14 and Rmk. 1.15 to show many cases in which the hypothesis of Thm. 3.3 are fulfilled.

To illustrate our results, we give throughout the paper examples of groups that cannot be Kähler, most of them previously unknown to the author.

Acknowledgements: The author wishes to thank V. Navarro Aznar, for originally proposing the problem and many interesting discussions on it. Manfred Hartl for his valuable help in $\S 1, F$. Campana and the members of the Cigrons Seminar of the Universitat de Barcelona are also to be thanked.

\section{Nilpotent Lie algebras related to a group}

We will recall here the concept of Malcev completion of a group and some related nilpotent Lie algebras $\mathcal{L}_{n} G$. We also give an algorithm to compute $\mathcal{L}_{2} G$ derived from $[S t]$. 
Let $G$ be a finitely presented group. We can functorially assign to it a tower of nilpotent groups

$$
\cdots \rightarrow \Gamma_{1} / \Gamma_{3} G \rightarrow \Gamma_{1} / \Gamma_{2} G \rightarrow 1
$$

where $\Gamma_{1} G=G, \Gamma_{n} G=\left[\Gamma_{n-1} G, G\right]$ and $\Gamma_{1} / \Gamma_{n} G=G / \Gamma_{n} G$.

A group $G$ is said to be uniquely divisible when for any pair $g \in G, n \in \mathbb{Z}, g$ has a unique $n$th root in $G$. The category $n-\mathbb{Q}-\mathcal{G} r$ of uniquely divisible nilpotent groups is included in the category $n-\mathcal{G} r$ of nilpotent groups, and the inclusion functor has a left adjoint, the Malcev completion functor $\otimes \mathbb{Q}: n-\mathcal{G} r \rightarrow n-\mathbb{Q}-\mathcal{G} r$. The functor $\otimes \mathbb{Q}$ is the ordinary tensor product on abelian groups. For two alternative ways of defining the Malcev completion, see [HMR] Part I, or App. A of [Q].

The Baker-Campbell-Hausdorff formula gives a categorical equivalence between finitely generated groups in $n-\mathbb{Q}-\mathcal{G} r$ and finite-dimensional nilpotent $\mathbb{Q}$-Lie algebras. In the latter category there are $\otimes \mathbb{R}, \otimes \mathbb{C}$ functors, and crossing back and forth in this manner we may define a $\otimes \mathbb{R}$ functor over $n-\mathcal{G} r$. Thus we naturally associate to $G$ a tower

$$
\cdots \rightarrow \Gamma_{1} / \Gamma_{3} G \otimes \mathbb{R} \rightarrow \Gamma_{1} / \Gamma_{2} G \otimes \mathbb{R} \rightarrow 1
$$

of uniquely divisible nilpotent Lie groups, and its correponding tower

$$
\cdots \rightarrow \mathcal{L}_{2} G \rightarrow \mathcal{L}_{1} G \rightarrow 0
$$

of nilpotent $\mathbb{R}$-Lie algebras.

Denote the lower central series of a Lie algebra $\mathcal{L}$ as $\mathcal{L}^{(1)}=\mathcal{L}, \mathcal{L}^{(n)}=\left[\mathcal{L}^{n-1}, \mathcal{L}\right]$. There is another tower of nilpotent $\mathbb{R}$-Lie algebras naturally associated to a group $G: \operatorname{Gr}_{n} G \otimes \mathbb{R}=\bigoplus_{i=1}^{n} \Gamma_{i} / \Gamma_{i+1} G \otimes \mathbb{R}$, with bracket induced by the group bracket. We sum up the properties of the tower of Lie algebras (1.1) that we will apply (see [L], [MKS] $\S 5,[S]$ LA $\S 4)$ :

\section{Proposition 1.2.}

(i) The Lie algebras $\mathcal{L}_{n} G$ have nilpotency class nil $\mathcal{L}_{n} G=n$.

(ii) The tower maps $\mathcal{L}_{n+1} G \rightarrow \mathcal{L}_{n} G$ induce isomorphisms $\mathcal{L}_{n+1} G / \mathcal{L}_{n+1} G^{(n+1)} \cong$ $\mathcal{L}_{n} G$.

(iii) There are isomorphisms of $\mathbb{R}$-vector spaces $\mathcal{L}_{n}^{(n)} G \cong \Gamma_{n} / \Gamma_{n+1} G \otimes \mathbb{R}$.

(iv) The graduation of $\mathcal{L}_{n} G$ by its lower central series produces a natural tower of isomorphisms of graded Lie algebras $\mathcal{L}_{n} G \cong G r \mathcal{L}_{n} G \cong G r_{n} G \otimes \mathbb{R}$.

We will call the Malcev algebra and denote $\mathcal{L} G$ the pronilpotent algebra $\lim \mathcal{L}_{n} G$, which is equivalent to the Malcev completion $G \otimes \mathbb{R}$ by the BakerCampbell-Hausdorff formula.

When the group $G$ is the fundamental group of a topological space $X$, the abelian algebra $\mathcal{L}_{1} G$ is just $H_{1}(X ; \mathbb{R})$. We will consider in this note the following simplest algebra, $\mathcal{L}_{2} G \cong \Gamma_{1} / \Gamma_{2} G \otimes \mathbb{R} \oplus \Gamma_{2} / \Gamma_{3} G \otimes \mathbb{R}$. The algebra $\mathcal{L}_{2} G$ is the quotient of the Malcev algebra $\mathcal{L} G$ by its third commutator ideal $\mathcal{L} G^{(3)}$, and is also the quotient of the holonomy algebra of $G \mathfrak{g}_{G}$ (cf. [Ch],[Ko]) by its third commutator ideal. 
The groups $G$ we will study will be given by finite presentations $G=\left\langle x_{1}, \ldots, x_{n}\right.$; $\left.r_{1}, \ldots, r_{s}\right\rangle$. This means that $G$ is defined by

$$
1 \longrightarrow N \longrightarrow F \longrightarrow G \longrightarrow 1
$$

where $F$ is the free group generated by the generator set $\left\{x_{1}, \ldots, x_{n}\right\}$, and $N$ is the normal subgroup of $F$ spanned by the relation set $\left\{r_{1}, \ldots, r_{s}\right\} \subset F$.

We describe the above constructions in a case which is fundamental for our purposes:

Example 1.4. Free groups.

Let $G=F_{n}=F_{\left\{x_{1}, \ldots, x_{n}\right\}}$. Its Malcev completion and Lie algebras $\mathcal{L}_{m} F_{n}$ may be computed by means of its group algebra (cf. [MKS],[Q],[S]). The conclusion is that, denoting by $\mathcal{L}(S)$ the free $\mathbb{R}$-Lie algebra spanned by a set $S$, there are isomorphisms

$$
\mathcal{L}_{m} F_{n} \cong \mathcal{L}\left(\left\{X_{1}, \ldots, X_{n}\right\}\right) / \mathcal{L}\left(\left\{X_{1}, \ldots, X_{n}\right\}\right)^{(m+1)}
$$

In particular, $\Gamma_{1} / \Gamma_{2} F_{n} \otimes \mathbb{R} \cong \mathbb{R} x_{1} \oplus \cdots \oplus \mathbb{R} x_{n}, \Gamma_{2} / \Gamma_{3} F_{n} \otimes \mathbb{R} \cong \mathbb{R}\left(x_{1}, x_{2}\right) \oplus \cdots \oplus$ $\mathbb{R}\left(x_{n-1}, x_{n}\right)$, and the brackets in $\mathcal{L}_{2} F_{n}$ are the group ones in $\Gamma_{1} / \Gamma_{2} F_{n}$ and zero all others.

The Lie algebra $\mathcal{L}_{2} G$ for a finitely presented $G$ may be obtained from its presentation and $\mathcal{L}_{2} F$. We will use an algorithm for computing them derived from [St], where a spectral sequence that computes all $J_{G}^{m} / J_{G}^{m+1}$ is described, and communicated to the author by Manfred Hartl.

Consider a group presentation $G=\left\langle x_{1}, \ldots, x_{n} ; r_{1}, \ldots, r_{s}\right\rangle$, which induces the exact sequence given in (1.3). Let $\mathbb{R} F, \mathbb{R} G$ be the $\mathbb{R}$-group algebras of $F, G$, and denote by $J_{F}, J_{G}$ their respective augmentation ideals. The sequence (1.3) induces an exact sequence of $\mathbb{R}$-algebras

$$
0 \longrightarrow K \longrightarrow \mathbb{R} F \longrightarrow \mathbb{R} G \longrightarrow 0
$$

where $K$ is the two-sided ideal generated by the $\mathbb{R}$-vector space $D=\left\langle r_{1}-1, \ldots, r_{s}-\right.$ 1) $\subset J_{F}$. This sequence restricts to exact sequences

$$
0 \longrightarrow K \longrightarrow J_{F}^{m}+K \longrightarrow J_{G}^{m} \longrightarrow 0
$$

for all $m \geq 1$. We will compute $J_{G} / J_{G}^{2}, J_{G}^{2} / J_{G}^{3}$ from those sequences:

Proposition 1.7. Consider the linear map $f: \bigoplus_{i=1}^{s} \mathbb{R} r_{i} \longrightarrow J_{F}$ determined by $r_{i} \mapsto r_{i}-1$.

(i) Let $d_{0}: \oplus \mathbb{R} r_{i} \rightarrow J_{F} / J_{F}^{2}$ be the projection of $f$. Then coker $d_{0} \cong J_{G} / J_{G}^{2}$.

(ii) The map $f$ induces a linear map

$$
\begin{aligned}
d_{1}: \operatorname{ker} d_{0} & \longrightarrow J_{F}^{2} /\left(J_{F}^{3}+J_{F} \cdot D+D \cdot J_{F}\right) \\
\sum \lambda_{i} r_{i} & \longmapsto \sum \lambda_{i}\left(r_{i}-1\right)
\end{aligned}
$$


and cokerd $d_{1} \cong J_{G}^{2} / J_{G}^{3}$.

Proof. (i) The exact sequences of (1.6) induce an isomorphism $J_{G} / J_{G}^{2} \cong J_{F} / J_{F}^{2}+K$. As $K$ is the two-sided ideal spanned by $D$ and $\mathbb{R} F \cong \mathbb{R} \oplus J_{F}$, actually $J_{F}^{2}+K=$ $J_{F}^{2}+D$, and thus $J_{G} / J_{G}^{2} \cong J_{F} / J_{F}^{2}+D$. By its construction, Im $d_{0}=D$, and this proves (i).

(ii) Again by (1.6) we have

$$
J_{G}^{2} / J_{G}^{3} \cong\left(J_{F}^{2} /\left(J_{F}^{2} \cap K\right)\right) /\left(J_{F}^{3} /\left(J_{F}^{3} \cap K\right)\right) \cong J_{F}^{2} /\left(J_{F}^{3}+J_{F}^{2} \cap K\right)
$$

The last denominator is $J_{F}^{3}+J_{F}^{2} \cap K=J_{F}^{3}+J_{F} \cdot D+D \cdot J_{F}+D \cap J_{F}^{2}$. Obviously $f\left(\operatorname{ker} d_{0}\right) \subset J_{F}^{2}$ and thus $d_{1}$ is well defined. Moreover, its image is precisely $D \cap J_{F}^{2}$, and (ii) follows from this.

We now relate the computed modules $J_{G} / J_{G}^{2}, J_{G}^{2} / J_{G}^{3}$ with the sought ones $\Gamma_{1} / \Gamma_{2}$, $\Gamma_{2} / \Gamma_{3} G \otimes \mathbb{R}$ applying a theorem by D. Quillen ([Q2]):

Theorem. Let $G$ be a group, $k$ a field of characteristic zero, $k G$ the group algebra and $j: \oplus \Gamma_{n} / \Gamma_{n+1} G \otimes k \rightarrow \oplus J_{G}^{n} / J_{G}^{n+1}$ given by $g \mapsto g-1$ over the homogeneous components.

Then $j$ induces an isomorphism of algebras $U\left(\oplus \Gamma_{n} / \Gamma_{n+1} G \otimes \mathbb{R}\right) \cong \oplus J_{G}^{n} / J_{G}^{n+1}$.

We may define the wedge product, or alternating product, of the associative algebra $\mathbb{R} G$ as

$$
x \wedge y:=x y-y x
$$

The wedge product of two linear subspaces $A, B \subset \mathbb{R} G$ is the linear subspace

$$
A \wedge B=\left\{w=\sum \lambda_{i} a_{i} \wedge b_{i} \in \mathbb{R} G \mid a_{i} \in A, b_{i} \in B, \lambda_{i} \in \mathbb{R}\right\}
$$

Quillen's theorem implies that the Lie algebra $\oplus \Gamma_{n} / \Gamma_{n+1} G \otimes \mathbb{R}$ is contained in the $J_{G}$-adic graduate of the group algebra, $\oplus J_{G}^{n} / J_{G}^{n+1}$. This inclusion sends the brackets of the Lie algebra to wedge products in $\oplus J_{G}^{n} / J_{G}^{n+1}$. In the cases $n=1,2$ this means:

Corollary 1.8.

(i) $\Gamma_{1} / \Gamma_{2} G \otimes \mathbb{R} \cong J_{G} / J_{G}^{2}$.

(ii) Consider the inclusion $J_{G} \wedge J_{G} \hookrightarrow J_{G}^{2}$. Then

$$
\Gamma_{2} / \Gamma_{3} G \otimes \mathbb{R} \cong\left(J_{G} \wedge J_{G}+J_{G}^{3}\right) / J_{G}^{3} \subset J_{G}^{2} / J_{G}^{3}
$$

Corollary 1.8 allows us to adapt the algorithm of Prop. 1.7 to compute $\Gamma_{1} / \Gamma_{2}$, $\Gamma_{2} / \Gamma_{3} G \otimes \mathbb{R}:$

Lemma 1.9. The image of the restriction $f: \operatorname{ker} d_{0} \rightarrow J_{F}^{2}$ lies in $J_{F} \wedge J_{F}+J_{F}^{3} \subset$ $J_{F}^{2}$.

Proof. Denote $F_{s}$ the free group generated by $\left\{y_{1}, \ldots, y_{s}\right\}$, and the map $r: F_{s} \rightarrow F$ sending $y_{i}$ to $r_{i}$. The map $d_{0}: \oplus \mathbb{R} r_{i} \rightarrow J_{F} / J_{F}^{2} \cong \Gamma_{1} / \Gamma_{2} F \otimes \mathbb{R}$ is the map induced 
by $r, \Gamma_{1} / \Gamma_{2}(r) \otimes \mathbb{R}: \Gamma_{1} / \Gamma_{2} F_{s} \otimes \mathbb{R} \rightarrow \Gamma_{1} / \Gamma_{2} F \otimes \mathbb{R}$. Furthermore ker $\left(\Gamma_{1} / \Gamma_{2}(r) \otimes \mathbb{R}\right) \cong$ $\operatorname{ker}\left(\Gamma_{1} / \Gamma_{2}(r)\right) \otimes \mathbb{R}$, as $\Gamma_{1} / \Gamma_{2} F_{s}$ is a free abelian group. Thus ker $d_{0}$ admits a basis $\bar{w}_{1}, \ldots, \bar{w}_{k}$, with the $w_{i}$ words in $F_{s}$ mapping to $\Gamma_{2} F$ by $r$.

Now, the map $\Gamma_{2} F \rightarrow J_{F}^{2}$ sends a bracket $(a, b)$ to $(a-1)(b-1)-(b-1)(a-$ $1)+$ terms in $J_{F}^{3}$, and a product $\prod\left(a_{i}, b_{i}\right)$ to $\sum\left(a_{i}-1\right)\left(b_{i}-1\right)-\left(b_{i}-1\right)\left(a_{i}-1\right)+$ terms in $J_{F}^{3}$. Therefore, all the $w_{i}=\prod\left(a_{j_{i}}, b_{j_{i}}\right)$ map to $J_{F} \wedge J_{F}+J_{F}^{3}$.

Lemma 1.9 allows us to define a map $d_{1}: \operatorname{ker} d_{0} \rightarrow \bigwedge^{2}\left(\Gamma_{1} / \Gamma_{2} G \otimes \mathbb{R}\right)$ by composing

$$
\operatorname{ker} d_{0} \longrightarrow\left(J_{F} \wedge J_{F}+J_{F}^{3}\right) / J_{F}^{3} \cong \bigwedge^{2}\left(\Gamma_{1} / \Gamma_{2} F \otimes \mathbb{R}\right) \longrightarrow \bigwedge^{2}\left(\Gamma_{1} / \Gamma_{2} G \otimes \mathbb{R}\right)
$$

Proposition 1.10. coker $d_{1} \cong \Gamma_{2} / \Gamma_{3} G \otimes \mathbb{R}$.

Proof. As we have previously explained, $\Gamma_{1} / \Gamma_{2} G \otimes \mathbb{R} \cong J_{G} / J_{G}^{2} \cong J_{F} /\left(J_{F}^{2}+K\right) \cong$ $J_{F} /\left(J_{F}^{2}+D\right)$. Thus $\wedge^{2}\left(\Gamma_{1} / \Gamma_{2} G \otimes \mathbb{R}\right) \cong\left(J_{F} \wedge J_{F}+\left(J_{F}^{3}+J_{F} \cdot D+D \cdot J_{F}\right)\right) /\left(J_{F}^{3}+\right.$ $\left.J_{F} \cdot D+D \cdot J_{F}\right)$. Also $f\left(\operatorname{ker} d_{0}\right)=D \cap J_{F}^{2} \subset J_{F} \wedge J_{F}+J_{F}^{3}$ by Lemma 1.9, so

coker $d_{1} \cong\left(J_{F} \wedge J_{F}+J_{F}^{3}+J_{F} \cdot D+D \cdot J_{F}+D \cap J_{F}^{2}\right) /\left(J_{F}^{3}+J_{F} \cdot D+D \cdot J_{F}+D \cap J_{F}^{2}\right)$ $\cong\left(J_{F} \wedge J_{F}+J_{F}^{3}+K \cap J_{F}^{2}\right) /\left(J_{F}^{3}+K \cap J_{F}^{2}\right) \cong\left(J_{G} \wedge J_{G}+J_{G}^{3}\right) / J_{G}^{3} \cong \Gamma_{2} / \Gamma_{3} G \otimes \mathbb{R}$

the last isomorphism being given by Cor. 1.8 .

Corollary 1.11. $\operatorname{dim} \Gamma_{2} / \Gamma_{3} G \otimes \mathbb{R}=\left(\begin{array}{c}\operatorname{dim} \Gamma_{1} / \Gamma_{2} G \otimes \mathbb{R} \\ 2\end{array}\right)-\operatorname{dim} \operatorname{ker} d_{0}+\operatorname{dim} \operatorname{ker} d_{1}$

We are now able to determine the structure of the 2-step nilpotent Lie algebra $\mathcal{L}_{2} G$ of a finitely presented group $G=\left\langle x_{1}, \ldots, x_{n} ; r_{1}, \ldots, r_{s}\right\rangle$ :

Proposition 1.12. Let $\bigwedge^{\leq 2}\left(\Gamma_{1} / \Gamma_{2} G \otimes \mathbb{R}\right)$ be the free exterior algebra generated by $\Gamma_{1} / \Gamma_{2} G \otimes \mathbb{R}$ modulo the ideal $\wedge^{\geq 3}\left(\Gamma_{1} / \Gamma_{2} G \otimes \mathbb{R}\right)$ generated by wedges of length 3 or more. There is an isomorphism

$$
\mathcal{L}_{2} G \cong\left(\bigwedge^{\leq 2}\left(\Gamma_{1} / \Gamma_{2} G \otimes \mathbb{R}\right)\right) /\left(\operatorname{ker} d_{0} / \operatorname{ker} d_{1}\right)
$$

Proof. There is an obvious map of exterior algebras, which is a linear isomorphism in every degree by the above results.

Thus $\mathcal{L}_{2} G$ is the quotient of a free 2 -step nilpotent $\mathbb{R}$-Lie algebra $\bigwedge^{\leq 2}\left(H_{1}(G ; \mathbb{R})\right)$ by a subspace of 2 -brackets ker $d_{0} / \operatorname{ker} d_{1}$, which corresponds to the relations of the holonomy algebra. We have stated in Ex. 1.4 the case of free groups. Let us examine this structure in some other simple cases:

Corollary 1.13. Let $G=\left\langle x_{1}, \ldots, x_{n} ; r\right\rangle$ be a group admitting a presentation with a single relation. Then:

(i) If $r \notin \Gamma_{2} F$, there is an isomorphism $\mathcal{L}_{2} G \cong \mathcal{L}_{2} F_{n-1}$ with $F_{n-1}$ a free group of rank $n-1$. 
(ii) If $r \in \Gamma_{2} F \backslash \Gamma_{3} F$, there is an isomorphism $\mathcal{L}_{2} G \cong \mathcal{L}_{2} F / d_{1}(r)$.

(iii) If $r \in \Gamma_{3} F$, there is an isomorphism $\mathcal{L}_{2} G \cong \mathcal{L}_{2} F$.

Proof. All cases are found by applying Prop. 1.12.

(i) In this case $\Gamma_{1} / \Gamma_{2} G \otimes \mathbb{R} \cong \Gamma_{1} / \Gamma_{2} F_{n-1} \otimes \mathbb{R}$, and as $r \notin \Gamma_{2} F$, ker $d_{0}=\{0\}$.

(ii) In this case the map $F \rightarrow G$ induces an isomorphism $\Gamma_{1} / \Gamma_{2} F \otimes \mathbb{R} \cong \Gamma_{1} / \Gamma_{2} G \otimes \mathbb{R}$, ker $d_{0}=\mathbb{R} r$, and as $r \notin \Gamma_{3} F$, the coincidence of the lower central series and augmentation ideal power filtrations in free groups ([MKS] 5.12,[S]) shows that $r-1 \notin J_{F}^{3}$, hence $d_{1}(r) \neq 0$.

(iii) In this case, ker $d_{0}=\mathbb{R} r$ and again by the above coincidence of filtrations, $d_{1}(r)=0$.

Corollary 1.14. Let $G=\left\langle x_{1}, \ldots, x_{n} ; r_{1}, \ldots, r_{s}\right\rangle$ be a finitely presented group such that its defining relations may be divided in two sets: $\left\{r_{1}, \ldots, r_{k}\right\}$ such that $\bar{r}_{1}, \ldots, \bar{r}_{k}$ are linearly independent in $\Gamma_{1} / \Gamma_{2} F \otimes \mathbb{R}$ and $\left\{r_{k+1}, \ldots, r_{s}\right\}$ which belong to $\Gamma_{3} F$. Then there is an isomorphism $\mathcal{L}_{2} G \cong \mathcal{L}_{2} F_{n-k}$, where $F_{n-k}$ is a free group of rank $n-k$.

Proof. In this case $\Gamma_{1} / \Gamma_{2} G \otimes \mathbb{R}$ has rank $n-k$, ker $d_{0}=\mathbb{R} r_{k+1} \oplus \cdots \oplus \mathbb{R} r_{n}$ because those $r_{j}$ are commutators and the other relations form a basis of $\operatorname{Im} f$, and ker $d_{1}=$ ker $d_{0}$ because $r_{k+1}, \ldots, r_{n} \in \Gamma_{3} F$.

Remark 1.15. We will be interested in this note in which groups $G$ have a free 2-step nilpotent Lie algebra $\mathcal{L}_{2} G$, which by Prop. 1.12 is equivalent to ker $d_{0}=\operatorname{ker} d_{1}$.

Generic presentations with less relations than generators produce a free $\mathcal{L}_{2} G$. The reason is that given a group presentation $G=\left\langle x_{1}, \ldots, x_{n} ; r_{1}, \ldots, r_{s}\right\rangle$ with a number of relations $s \leq n$, ker $d_{0}=0$ and therefore $\mathcal{L}_{2} G$ is free, unless the classes $\bar{r}_{1}, \ldots, \bar{r}_{s} \in \Gamma_{1} / \Gamma_{2} F_{n} \otimes \mathbb{R}$ are linearly dependent. But the sets of linearly dependent $\bar{r}_{1}, \ldots, \bar{r}_{s}$ form a codimension $n-s+1$ closed subset of $\left(\Gamma_{1} / \Gamma_{2} F_{n} \otimes \mathbb{R}\right)^{s}$.

The hypotheses of Corollary 1.14 may be weakened by requiring only that $\left\{r_{1}, \ldots, r_{k}\right\}$ map on a basis of $\operatorname{Im} d_{0}$, and the remaining relations $\left\{r_{k+1}, \ldots, r_{s}\right\}$ belong to $\Gamma_{3} F \cdot N_{k}$, where $N_{k}$ is the normal closure in $F$ of $\left\{r_{1}, \ldots, r_{k}\right\}$.

\section{§2. Sullivan's 1-minimal models, brackets and cup products}

We sum up for the reader's convenience some basic facts on Sullivan's 1-minimal models, its equivalence with the Malcev completion of the fundamental group and its relation with cup products.

Let $X$ be now a differentiable manifold, and $\mathcal{E}^{*}(X)$ its De Rham complex.

The theory of minimal models developed by Sullivan shows that $\mathcal{E}^{*}(X)$ has a 1minimal model. This is a certain minimal commutative differential graded algebra (cdga) $M(2,0)(X)$, defined as the limit of an inductive system of cdga $M(1,1) \hookrightarrow$ $M(1,2) \hookrightarrow M(1,3) \hookrightarrow \ldots$, together with an algebra morphism $\rho: M_{X} \rightarrow \mathcal{E}^{*}(X)$ such that $H^{0}(\rho), H^{1}(\rho)$ are isomorphisms and $H^{2}(\rho)$ is a monomorphism. For a 
more complete and elementary account of the theory of minimal models we remit ourselves to $[\mathrm{GM}]$, which treats 1 -minimal models in Chap. XII. We will only review the construction up to the second step $M(1,2)$, which will be used to relate $\pi_{1}(X, *)$ and cup products on $H^{1}(X)$.

Define $M(1,1)=\bigwedge\left(V_{1}^{1}\right)$, the free exterior algebra generated by the $\mathbb{R}$-vector space $V_{1}^{1}=H^{1}(X)$. Every element of $V_{1}^{1}$ is defined to have degree one and boundary zero, and the map $\rho: M(1,1) \rightarrow \mathcal{E}^{*}(X)$ sends every $x \in V_{1}^{1}$ to its image in a prefixed

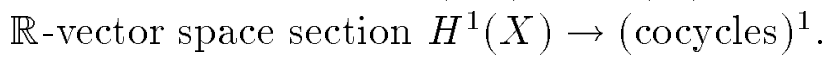

The (1,2)-minimal model is defined as an extension of $M(1,1): M(1,2)=\bigwedge\left(V_{1}^{1} \oplus\right.$ $\left.V_{2}^{1}\right)$, where $V_{2}^{1}=\operatorname{ker}\left(H^{2} \rho: H^{2} M(1,1) \rightarrow H^{2} \mathcal{E}^{*}(X)\right)$. For any $v \in V_{2}^{1}$ we define $d v$ as the element of $\bigwedge^{2} V_{1}^{1}$ defining its cohomology class, and if $d v=\sum x_{i} y_{i}, \rho(v)$ is a linearly varying primitive of $\sum \rho\left(x_{i}\right) \rho\left(y_{i}\right)$ in $\mathcal{E}^{*}(X)$.

Remark 2.1. By its definition, $H^{2} M(1,1)$ is the exterior product $\wedge^{2} H^{1}(X)$, and as $\rho$ is a cdga morphism, there is an isomorphism $V_{2}^{1}=\left(\operatorname{ker} H^{2} M(1,1) \rightarrow H^{2} \mathcal{E}^{*}(X)\right) \cong$ $\operatorname{ker}(\cup$ :

$\bigwedge^{2} H^{1}(X) \rightarrow H^{2}(X)$.

The following steps $M(1, n)$ are constructed in a likewise manner, defining $V_{n}^{1}$ as ker $H^{2} M(1, n-1) \rightarrow H^{2} \mathcal{E}^{*}(X)$, and $d, \rho$ on it as on $V_{2}^{1}$. The inductive limit is denoted $M(2,0)$ and is the 1-minimal model of $X$.

Remark 2.2. Among the properties of the 1-minimal model let us remark:

- It is well defined up to isomorphism.

- It is functorial up to homotopy, i.e., any cdga morphism $\mathcal{E}^{*} Y \rightarrow \mathcal{E}^{*} X$ may be lifted to a morphism $M(2,0)(Y) \rightarrow M(2,0)(X)$, and all its liftings are homotopic in the cdga category.

- As a consequence of its uniqueness, if a map $f: X \rightarrow Y$ induces isomorphisms $H^{0} f, H^{1} f$ and a monomorphism $H^{2} f$, then it induces an isomorphism of 1 minimal models $M(2,0)(Y) \cong M(2,0)(X)$.

- The 1-minimal model of $X$ may be computed replacing $\mathcal{E}^{*}(X)$ by any other cdga quasi-isomorphic to it. Thus if $X$ is a complex manifold, we may compute it from its holomorphic De Rham complex, logarithmic complexes, Dolbeault complexes, etc..

We recall now the dualizing process between Lie algebras and free commutative differential graded algebras generated by elements of degree one.

Let $L$ be a finite-dimensional $\mathbb{R}$-Lie algebra. Its bracket is a bilinear alternating map

$$
[., .]: \bigwedge^{2} L \longrightarrow L
$$

Dualizing on both sides, $[.,$.$] has an adjoint map$

$$
d: L^{\vee} \longrightarrow \bigwedge^{2} L^{\vee}
$$


The map $d$ may be extended as a graded derivation to the free graded algebra $\bigwedge L^{\vee}$, defining the degree of elements in $V^{\vee}$ to be one. The Jacobi identity satisfied by $[.,$.$] dualizes then as d^{2}=0$.

Reciprocally, if $M=\bigwedge W$ is a free cdga and $\operatorname{deg} W=1$, the differential restricts to a map $d: W=M^{1} \rightarrow M^{2}=\bigwedge^{2} W$, which dualizes to a map [...]: $\bigwedge^{2} W^{\vee} \rightarrow$ $W^{\vee}$, and the fact $d^{2}=0$ in $M$ translates as the Jacobi identity in $W^{\vee}$.

Definition 2.5. A Lie algebra $L$ and a free cdga generated by elements of degree one are dual when each one yields the other by the above processes.

The following result is due to D. Sullivan. The reader will find a complete proof of it in $[B G]$.

Theorem 2.6. (Sullivan) Let $X$ be an arc-connected topological space with a finitely presented fundamental group $\pi_{1}(X, *)$. The inductive system $M(1,1) \hookrightarrow$ $M(1,2) \hookrightarrow \ldots$ formed by the $(1, n)$-minimal models of $X$ and the projective system $\cdots \rightarrow \mathcal{L}_{2} \pi_{1} X \rightarrow \mathcal{L}_{1} \pi_{1} X$ described in (1.1) are dual.

This theorem has important consequences for our purposes. The most obvious is about the duality as vector spaces:

Corollary 2.7. $V_{n}^{1} \cong\left(\Gamma_{n} / \Gamma_{n+1}\left(\pi_{1} X\right) \otimes \mathbb{R}\right)^{\vee}$

The duality Lie algebra-cdga also has consequences:

Lemma 2.8. The diagram

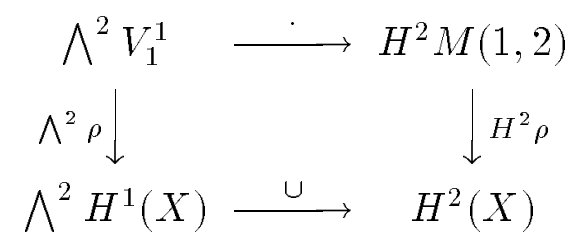

is commutative, and the first column is an isomorphism.

Remark 2.9. Lemma 2.8 implies that the cup product between 1-classes is determined by the brakets in $\mathcal{L}_{2} \pi_{1} X$. This is due to the fact that it factors through $M(1,2)$, which is dual to $\mathcal{L}_{2} \pi_{1} X \hookrightarrow \mathcal{L}_{2} \pi_{1} X$.

Another particular consequence of Theorem 2.6 we will use is:

Corollary 2.10. $\operatorname{dim} \operatorname{ker}\left(\cup: \bigwedge^{2} H^{1}(X) \rightarrow H^{2}(X)\right)=\operatorname{dim} \mathcal{L}_{2} \pi_{1} X^{(2)}=\operatorname{dim} \Gamma_{2} / \Gamma_{3} \pi_{1}(X, *) \otimes$ $\mathbb{R}$

Proof. Both spaces are isomorphic to $V_{2}^{1}$.

\section{$\S 3$. The $\mathcal{L}_{2}$ algebras of Kähler groups}

In this chapter we prove that groups with a free $\mathcal{L}_{2}$ algebra cannot be Kähler (Thm. 3.3), which by Remark 1.15 is the generic situation in groups with few defining relations. 
We recall now the definition of the objects of our interest:

Definition 3.1. Let $G$ be a group. It is a Kähler group when $G \cong \pi_{1}(X, *)$, where $X$ is a compact Kähler manifold.

Some restrictions on Kähler groups are well known: They are finitely presented, their rank is even. Sullivan's theory of minimal models, completed by Morgan, ... , yields subtle conditions Kähler groups must satisfy, due to the formality of compact Kähler manifolds ([DGMS]), and to the mixed Hodge structure its 1-minimal model supports, which is an extension of the Hodge structure on the cohomology ring ([M1],[M2]). The formality condition it imposes is in our case:

Proposition 3.2. ([M1],9.4) Let $G$ be a Kähler group. The Lie algebra Gr $\mathcal{L} G$ is the quotient of a free Lie algebra by an ideal generated by sums of length two brackets.

This is equivalent to the Malcev and holonomy algebras of $G$ being isomorphic (cf. $[\mathrm{Ko}]$ ). For instance, the group $G=\langle x, y ;((x, y), y)\rangle$ cannot be Kähler because $\mathcal{L} G$ is the quotient of a free algebra by the ideal generated by a length three bracket $[[\bar{x}, \bar{y}], \bar{y}]$.

In this note we will look in another direction through the results of the preceding section. Johnson and Rees rule out in [JR] free groups as Kähler groups because they have finite index subgroups of odd rank. In this paragraph we establish this by studying cup products, and in this way the result holds for groups with a free 2step nilpotent Lie algebra $\mathcal{L}_{2}$. This is a consequence of the Lefschetz decomposition and the nondegenerate alternating pairing $Q$ that the real cohomology $H^{*}(X)$ of a compact Kähler manifold $X$ supports. Our standard reference for these properties will be $[\mathrm{W}]$, Chap. V.

Theorem 3.3. Let $G$ be a finitely presented group such that $\mathcal{L}_{2} G \cong \mathcal{L}_{2} F_{n}$ for some n. Then $G$ is not Kähler.

Proof. Suppose $\pi_{1}(X, *) \cong G$. As $\mathcal{L}_{2} G \cong \mathcal{L}_{2} F_{n}$, and using Example 1.4, $\operatorname{dim} \Gamma_{1} / \Gamma_{2} G \otimes$ $\mathbb{R}=\operatorname{dim} \Gamma_{1} / \Gamma_{2} F_{n} \otimes \mathbb{R}=n$, and $\operatorname{dim} \Gamma_{2} / \Gamma_{3} G \otimes \mathbb{R}=\operatorname{dim} \mathcal{L}_{2} G^{(2)}=\operatorname{dim} \mathcal{L}_{2} G^{(2)}=$ $\operatorname{dim} \mathcal{L}_{2} F_{n}=\frac{n(n-1)}{2}$. Thus by Cor. 2.10 ker $U=\wedge^{2} H^{1}(X)$, all cup products are zero. But cohomology classes of rank one are primitive, and the pairing $Q$ is nondegenerate (see [W],5.6). This leads to a contradiction, hence $G$ cannot be Kähler.

To use Theorem 3.3, one needs to know when $\mathcal{L}_{2} G \cong \mathcal{L}_{2} F_{n}$. This can be established from a presentation of $G$ as we have shown in section $\S 1$ (see Remark 1.15). We will now remark the case of one-relator groups. The consequence for them of [M1],9.4 is that if $G$ admits a presentation $G=\left\langle x_{1}, \ldots, x_{n} ; r\right\rangle$, then its single defining relation $r$ does not lie in $\Gamma_{3} F\left\{x_{1}, \ldots, x_{n}\right\}$. We complete this with

Corollary 3.4. Let $G=\left\langle x_{1}, \ldots, x_{n} ; r\right\rangle$ be a Kähler group presented with a single relation. Then $r \in \Gamma_{2} F\left\{x_{1}, \ldots, x_{n}\right\} \backslash \Gamma_{3} F\left\{x_{1}, \ldots, x_{n}\right\}$. 
Proof. By Corollary 1.13, $r \in \Gamma_{2} F \backslash \Gamma_{3} F$ is the only case in which $\mathcal{L}_{2} G$ is not free.

Examples 3.5.

(i) ([JR]) Free groups cannot be Kähler.

(ii) The group $G=\langle x, y ;((x, y), y)\rangle$ cannot be Kähler because $((x, y), y) \in \Gamma_{3} F$ and therefore $\mathcal{L}_{2} G \cong \mathcal{L}_{2} F_{2}$. This group was known to be non-Kähler by [M1],[M2].

(iii) The group $G=\left\langle x, y, z, t ; x^{3} y^{-4} z^{2} y, y^{2} z^{2}\right\rangle$ is not Kähler because the two defining relations are linearly independent in $\Gamma_{1} / \Gamma_{2} F \otimes \mathbb{R} \cong \mathbb{R}^{4}$, and thus by Cor. 1.14 $\mathcal{L}_{2} G \cong \mathcal{L}_{2} F_{2}$.

(iv) The group $G=\left\langle x_{1}, \ldots, x_{5} ; x_{1} x_{2}^{2} x_{1}, x_{2} x_{3}^{2} x_{2}, x_{5} x_{4}^{2} x_{5}\right\rangle$ has also a linearly independent relation set, and thus $\mathcal{L}_{2} G \cong \mathcal{L}_{2} F_{2}$, and $G$ cannot be Kähler either.

(v) Compact Riemann surfaces provide examples showing that one-relator groups with a defining relation $r \in \Gamma_{2} F \backslash \Gamma_{3} F$ are possible.

\section{$\S 4$. One- and two-relator Kähler groups}

In this chapter we give a lower bound for the number of defining relations that a presentation of a Kähler group $\pi_{1} X$ must have, determined by the dimension of the Albanese image of $X$ (Prop. 4.6). We apply this to fully determine the Malcev completions of one- and two-relator Kähler groups in Thm. 4.7. Our starting point 4.3 is due to $\mathrm{F}$. Campana $([\mathrm{C}])$.

The Albanese variety and Albanese map are another feature of compact complex manifolds which is very useful to study its fundamental group. Let us briefly recall it.

Proposition 4.1. Let $X$ be a compact Kähler manifold, and let $q=\operatorname{dim} H^{0}\left(\Omega_{X}^{1}\right)$. There is a complex torus $A l b(X)$ of dimension $q$ (the Albanese torus) and a holomorphic map $\alpha_{X}: X \rightarrow A l b(X)$ (the Albanese map) such that $\alpha_{X}$ induces an isomorphism $H^{1}(A l b(X) ; \mathbb{Z}) \stackrel{\cong}{\longrightarrow} H^{1}(X ; \mathbb{Z})$. The pair $\left(A l b(X), \alpha_{X}\right)$ is determined up to isomorphism by this property. Moreover $\alpha_{X}(X)$ is a generating set for $A l b(X)$ as an abelian group.

Let us fix our notation: Let $X$ be compact Kähler, $\alpha_{X}: X \rightarrow A l b(X)$ its Albanese map, and denote $Y=\alpha_{X}(X)$ its image, which may be singular. We consider a desingularization $\varepsilon: \tilde{Y} \rightarrow Y$, and a desingularization $\tilde{X}$ of the pullback of $\alpha_{X}$ :

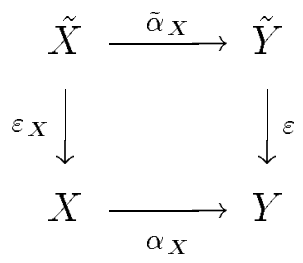

It is clear that the manifold $\tilde{X}$ is also compact Kähler, and that the map $\varepsilon_{X}$ induces an isomorphism $\varepsilon_{X *}: \pi_{1} \tilde{X} \rightarrow \pi_{1} X$. 
We will call the map $\tilde{\alpha}_{X}: \tilde{X} \rightarrow \tilde{Y}$ a smoothing of the Albanese map of X. The properties of the original Albanese map $\alpha_{X}$ relate $X, \tilde{X}$ and $\tilde{Y}$ for our purposes:

Proposition 4.3. ([C]) Let $X$ be compact Kähler, and $\tilde{\alpha}_{X}: \tilde{X} \rightarrow \tilde{Y}$ be a smoothing of the Albanese map of $X$. Then $\tilde{\alpha}_{X}$ induces an isomorphism $\mathcal{L}\left(\pi_{1} \tilde{X}\right) \stackrel{\sim}{\longrightarrow} \mathcal{L}\left(\pi_{1} \tilde{Y}\right)$.

Proof. (Cf. [C]) As $\varepsilon_{X}$ induces an isomorphism of fundamental groups, hence $\varepsilon_{X}^{*}: H^{1}(X) \rightarrow H^{1}(\tilde{X})$ is also an isomorphism. This implies that $A l b(X)$ is the Albanese torus of $\tilde{X}$ and $\alpha_{X} \circ \varepsilon_{X}=\varepsilon \circ \tilde{\alpha}_{X}$ its Albanese map. As a consequence $\tilde{\alpha}_{X}^{*}: H^{1}(\tilde{Y}) \rightarrow H^{1}(\tilde{X})$ is onto. As $\alpha_{X}$ itself is also onto, $\tilde{\alpha}_{X}^{*}$ is also injective for $H^{*}$. Therefore $\tilde{\alpha}_{X}$ induces an isomorphism $H^{1}(\tilde{Y}) \cong H^{1}(\tilde{X})$ and an injection $H^{2}(\tilde{Y}) \hookrightarrow H^{2}(\tilde{X})$. Thus by universality of the 1-minimal model, $\tilde{\alpha}_{X}$ induces an isomorphism $M(2,0)(\tilde{Y}) \cong M(2,0)(\tilde{X})$; our statement is its dual.

Thus the study of Malcev completions of Kähler groups may be reduced to the study of smoothings of its Albanese images. This is specially convenient in the following case:

Corollary 4.4. ([C]) Let $X$ be compact Kähler, with $q=\frac{1}{2} b_{1}(X)=1$. Then the Malcev algebra of $X$ is $\mathcal{L} \pi_{1}(X) \otimes \mathbb{R} \cong\left(\mathbb{R}^{2},+\right)$.

Proof. The Albanese torus $E=A l b(X)$ has dimension 1 in this case. Therefore $\alpha_{X}$ is onto, has smooth image and so equals $\tilde{\alpha}_{X}$.

The elliptic curve $E$ has abelian fundamental group $\pi_{1}(E) \cong \mathbb{Z}^{2}$, thus our statement is a direct consequence of Prop. 4.3.

But rather than follow this line, we will derive from it consequences on $H^{*}(X)$, which will determine Malcev completions of Kähler groups with one or two defining relations.

Lemma 4.5. Let $X$ be compact Kähler, $Y$ the Albanese image of $X$ and $m=$ $\operatorname{dim}_{\mathbb{C}} \tilde{Y}$. Then the graded algebra $H^{*}(X ; \mathbb{C})$ contains a free graded exterior algebra $\bigwedge(V)$, where $V$ is a complex vector space of dimension $m$ and degree 1 spanned by holomorphic forms.

Proof. (cf. [Be] V.18) Let $y \in A l b(X)$ be a regular point of the Albanese image $Y=\alpha_{X}(X)$. As $\operatorname{dim} Y=m$, there are local coordinates $u_{1}, \ldots u_{n}$ of $A l b(X)$ in a neighbourhood $U$ of $y$ such that $Y \cap U$ is defined as $u_{m+1}=0, \ldots, u_{n}=0$. The holomorphic forms $d u_{1}, \ldots, d u_{m}$ are defined on $U$ and, as $A l b(X)$ is parallelizable, the forms in $\bigwedge\left(d u_{1}, \ldots, d u_{m}\right)$ extend to global holomorphic forms on $A l b(X)$. Its direct image $\bigwedge\left(\alpha_{X}^{*} d u_{1}, \ldots, \alpha_{X}^{*} d u_{m}\right)$ defines a subalgebra of holomorphic cohomology classes in $H^{*}(X)$ which is free over $y$, hence is free.

The above Lemma together with the correspondence of 2.10 may be used to bound from below the number of defining relations for Kähler groups, and to study those admitting a one- or two-relation presentation. 
Proposition 4.6. Let $G$ be a Kähler group, $X$ a compact Kähler manifold such that $\pi_{1} X \cong G$ and $Y$ its Albanese image. Then:

(i) If $\operatorname{dim} Y=1$, there is an isomorphism $\mathcal{L} G \cong \mathcal{L} \pi_{1} C_{g}$ with $C_{g}$ a compact Riemann surface of genus $g$, induced by a group map $G \rightarrow \pi_{1} C_{g}$.

(ii) If $\operatorname{dim} Y=m>1$, $\operatorname{dim} \operatorname{ker}\left(d_{0}: \mathbb{R} r_{1} \oplus \cdots \oplus \mathbb{R} r_{s} \rightarrow \Gamma_{1} / \Gamma_{2} F \otimes \mathbb{R}\right) \geq 2\left(\begin{array}{c}m \\ 2\end{array}\right)+1$. In particular, any presentation $G=\left\langle x_{1}, \ldots, x_{n} ; r_{1}, \ldots, r_{s}\right\rangle$ must have defining relations $r_{1}, \ldots, r_{k}$ such that they form a basis of $\operatorname{Im} f$ and at least another $2\left(\begin{array}{c}m \\ 2\end{array}\right)+1$ defining relations.

\section{Proof.}

(i) is just Prop. 4.3 with $\tilde{Y}$ as $C_{g}$.

(ii) By Lemma 4.5, the algebra $H^{*}(X ; \mathbb{C})$ contains a free algebra $\bigwedge(V)$ generated by $m$ linearly independent holomorphic 1-forms. By the Hodge structure of $H^{*}(X)$ it contains an isomorphic algebra $\bigwedge(\bar{V})$ spanned by $m$ independent antiholomorphic 1-forms. Both algebras being free, one obtains the lower bound $\operatorname{dim} \operatorname{Im} \cup: \bigwedge^{2} H^{1}(X) \rightarrow H^{2}(X) \geq 2\left(\begin{array}{c}m \\ 2\end{array}\right)$ considering either holomorphic or antiholomorphic products alone. Finally, due to the properties of the $Q$ pairing in $H^{1}(X)$ ([W] 5.6), the product of a holomorphic 1-form with its conjugate cannot be zero, so $\operatorname{dim}(\operatorname{Im} U) \cap H^{1,1}(X) \geq 1$. By the correspondence of 2.10 this produces the sought bound.

Remark. If $X$ is compact Kähler and satisfies (i) in the above Prop. 4.6, it is not hard to check that the map $\pi_{1} X=G \rightarrow \pi_{1} C_{g}$ is onto. If the genus $g$ is $\geq 2$, such groups $G$ are examples of what we will call fibered Kähler groups, to be defined in 5.1 .

We are now able to complete our study of compact Kähler groups with one or two defining relations begun in Cor. 3.4.

Theorem 4.7. Let $G$ be a Kähler group admitting a presentation with only one or two defining relations. Then either $\Gamma_{1} / \Gamma_{2} G \otimes \mathbb{R}=0$ or $\mathcal{L} G \cong \mathcal{L} \pi_{1} C_{g}$ with $C_{g}$ a compact Riemann surface.

Proof. If $\Gamma_{1} / \Gamma_{2} G \otimes \mathbb{R} \neq 0$, then $\mathcal{L}_{2} G \neq 0$, and by Prop. 4.6 any presentation of $G$ must have at least $2\left({ }_{2}^{\operatorname{dim} Y}\right)+1$ defining relations, with $Y=A l b(X)$. Thus the only possible case is $\operatorname{dim} Y=1$, and Prop. 4.6 (i) completes the proof.

Remark.

- The 1-relator groups $G$ with $\Gamma_{1} / \Gamma_{2} G \otimes \mathbb{R}=0$ are exactly the $G \cong \mathbb{Z} / n \mathbb{Z}$.

- The 2-relator groups $G$ with $\Gamma_{1} / \Gamma_{2} G \otimes \mathbb{R}=0$ are those with a presentation $\left\langle x_{1}, x_{2} ; r_{1}, r_{2}\right\rangle$ with $\bar{r}_{1}, \bar{r}_{2}$ linearly independent in $\Gamma_{1} / \Gamma_{2} F\left\{x_{1}, x_{2}\right\}$.

This is immediately derived from the exact sequence (1.5).

Examples 4.8. Denote $C_{g}$ a compact Riemann surface of genus $g$.

(i) The group $G$ defined in Ex. 3.5 (iii) can also be seen not to be Kähler by Thm. 4.7 , as $\Gamma_{1} / \Gamma_{2} G \otimes \mathbb{R} \cong \mathbb{R}^{2}$ but $\mathcal{L}_{2} G \approx \mathcal{L}_{2} \pi_{1}\left(C_{1}\right)$.

(ii) The group $G=\left\langle x_{1}, x_{2}, x_{3}, x_{4} ;\left(x_{1} x_{2}, x_{3}^{2}\right),\left(x_{1} x_{3} x_{1}, x_{4}^{3}\right)\right\rangle$ has a Malcev algebra which fulfills the quadratic presentation condition imposed by Morgan (Prop. 
3.2). Yet $G$ cannot be Kähler because $\Gamma_{1} / \Gamma_{2} G \otimes \mathbb{R} \cong \mathbb{R}^{4}$ but $\operatorname{dim} \Gamma_{2} / \Gamma_{3} G \otimes \mathbb{R}=$ $4 \neq 5=\operatorname{dim} \Gamma_{2} / \Gamma_{3} \pi_{1}\left(C_{2}\right)$, contradicting Thm. 4.7.

\section{$\S 5$. Nonfibered Kähler groups}

Here we establish a dicothomy between fibered and nonfibered Kähler groups, arising from a result by A. Beauville and Y.T. Siu on the existence of irregular pencils on compact Kähler manifolds. We skip the fibered case, and we give in Prop. 5.6 an upper bound for $\operatorname{dim} \Gamma_{2} / \Gamma_{3} G \otimes \mathbb{R}$ in the case of nonfibered groups. This translates as a lower bound for the number of relations that their presentations must have.

Let $G=\pi_{1}(X, *)$ be a fundamental group. By Corollary $2.10 \operatorname{dim} \Gamma_{2} / \Gamma_{3} G \otimes \mathbb{R}=$ $\operatorname{dim} \bigwedge^{2} H^{1}(X)-\operatorname{dim} \operatorname{Im}\left(\cup: \bigwedge^{2} H^{1}(X) \rightarrow H^{2}(X)\right)$. We have seen in $\S 3$ that if $X$ is compact Kähler, Im $\cup$ must be nonzero. Now we will establish a lower bound on its dimension in the case of nonfibered manifolds, by recalling a result of CastelnuovoDe Franchis and its extension to arbitrary dimension.

Definition 5.1. Let $G$ be a Kähler group.

(i) We call $G$ a fibered Kähler group when $G=\pi_{1}(X, *)$ with $X$ compact Kähler admitting a nonconstant holomorphic map $f: X \rightarrow C_{g}$, with $C_{g}$ a compact Riemann surface of genus $g \geq 2$.

(ii) We call $G$ a nonfibered Kähler group when $G=\pi_{1}(X, *)$ with $X$ compact Kähler not admitting any nonconstant holomorphic map to a compact Riemann surface of genus $g \geq 2$.

A. Beauville and Y.T. Siu independently proved that the above definitions make sense:

Proposition 5.2. ([Be2], [Siu]) Let $X$ be a compact Kähler manifold, write $G=$ $\pi_{1}(X, *)$, and let $g \geq 2$ be an integer. Then $X$ admits a nonconstant holomorphic map to a compact Riemann surface of a genus $h \geq g$ if and only if there is an epimorphic group morphism $G \rightarrow \pi_{1}\left(C_{g}, *\right)$, with $\pi_{1}\left(C_{g}, *\right)$ the fundamental group of a compact Riemann surface of genus $g$.

Prop. 5.2 means that a Kähler group $G$ is either fibered or nonfibered, and that the former are characterised by admitting a $\pi_{1}\left(C_{g}\right)$ as a quotient.

Remark. If we have an onto map $G \rightarrow H \rightarrow 1$, it induces onto maps $\Gamma_{n} / \Gamma_{n+1} G \otimes$ $\mathbb{R} \rightarrow \Gamma_{n} / \Gamma_{n+1} H \otimes \mathbb{R} \rightarrow 0$ for all $n$. This together with the fact that the lower central series quotients of the $\pi_{1} C_{g}$ have all nonzero rank shows that nilpotent or rationally nilpotent Kähler groups must be nonfibered.

We now study the cup products of 1-forms in the case of nonfibered compact Kähler manifolds. We begin with an extension of a classical result (see [Ca]): 
Proposition 5.3. (Castelnuovo-De Franchis) Let $X$ be a compact Kähler manifold. If there exist $\omega_{1}, \omega_{2}$ linearly independent holomorphic 1-forms such that $\omega_{1} \wedge \omega_{2}=0$ then there is a holomorphic map $f: X \rightarrow C$ with $C$ a curve of genus $g(C) \geq 2$, such that $\omega_{1}, \omega_{2}$ belong to $\operatorname{Im} f^{*}$.

Remark. The form equality $\omega_{1} \wedge \omega_{2}=0$ is equivalent to $\omega_{1} \wedge \omega_{2}$ being exact. This is a result of Hodge theory, showing that a nonzero holomorphic form over a compact Kähler manifold cannot be exact.

The Castelnuovo-De Franchis theorem together with the conic structure of the set of products in $H^{2,0}(X)$ yield the following corollary (see [BPV] IV, Prop. 4.2):

Corollary 5.4. If $X$ is a nonfibered compact Kähler manifold, then $\operatorname{dim} \operatorname{Im}(\cup$ : $\left.\bigwedge^{2} H^{1,0}(X) \rightarrow H^{2,0}(X)\right) \geq 2 \operatorname{dim} H^{1,0}(X)-3$.

Cor. 5.4 gives a bound for the products of holomorphic 1-forms, and by conjugation, of antiholomorphic 1-forms. The dimension of products of holomorphicantiholomorphic 1-forms has been bounded for compact complex surfaces in [BPV], IV, Prop. 4.3. We slightly alter their proof to extend it to compact Kähler manifolds of arbitrary dimension:

Proposition 5.5. Let $X$ be a nonfibered compact Kähler manifold. Then $\operatorname{dim} \operatorname{Im}(\cup$ : $\left.H^{1,0}(X) \otimes H^{0,1}(X) \rightarrow H^{1,1}(X)\right) \geq 2 \operatorname{dim} H^{1,0}(X)-1$.

Proof. Denote $n=\operatorname{dim} X \geq 2, V=\operatorname{Im} \cup: H^{1,0}(X) \otimes H^{0,1}(X) \rightarrow H^{1,1}(X)$ and fix $\omega$ a fundamental Kähler form on $X$. We begin by showing that the pairing $\cup: H^{1,0}(X) \otimes H^{0,1}(X) \rightarrow V$ becomes injective when we fix a nonzero $\xi \in H^{1,0}(X)$ or $\bar{\eta} \in H^{0,1}(X)$.

Suppose there are holomorphic 1 -forms $\xi, \eta$ such that $\xi \wedge \bar{\eta}=d \alpha$. Then obviously $\xi \wedge \eta \wedge \bar{\xi} \wedge \bar{\eta}=d \alpha^{\prime}$, and

$$
\int_{X} \xi \wedge \eta \wedge \bar{\xi} \wedge \bar{\eta} \wedge \omega^{n-2}=0
$$

By the properties of the pairing $Q$ of compact Kähler manifolds (see [W] 5.6), this implies that $\xi \wedge \eta=0$, thus by the Castelnuovo-De Franchis theorem $\xi$ and $\eta$ are linearly dependent. Take $\xi=a \eta$, with $a \in \mathbb{C}^{*}$. Then $0=\xi \wedge \bar{\eta}=a \eta \wedge \bar{\eta}$. Again by the properties of the pairing $Q$, this means that $\xi, \eta=0$.

Thus a map may be defined

$$
\mathbb{P}\left(H^{1,0}(X)\right) \times \mathbb{P}\left(H^{0,1}(X)\right) \longrightarrow \mathbb{P}(V)
$$

with injective restrictions fixing a point in either factor of the source. We apply now the following result from $[\mathrm{RV}]$ :

Proposition. Let $\varphi: \mathbb{P}^{m}(\mathbb{C}) \times \mathbb{P}^{k}(\mathbb{C}) \rightarrow \mathbb{P}^{l}(\mathbb{C})$ be a holomorphic mapping, with $l<$ $m+k$. Then $\varphi$ factors through one of the projections $\mathbb{P}^{m} \times \mathbb{P}^{k} \rightarrow \mathbb{P}^{m}, \mathbb{P}^{m} \times \mathbb{P}^{k} \rightarrow \mathbb{P}^{k}$.

In our case, $U$ cannot factor through any of the projections because it is fiberwise injective in both cases, so it holds that $\operatorname{dim} V \geq 2 \operatorname{dim} H^{1,0}(X)-1$ as was wanted. 
We have now all the required pieces to study $\Gamma_{2} / \Gamma_{3} \otimes \mathbb{R}$ of nonfibered groups. We return to the notations defined in $\$ 1$.

Proposition 5.6. Let $X$ be a nonfibered compact Kähler manifold with $q=\frac{1}{2} \mathrm{dim}$ $\left.H^{1}(X)=\operatorname{dim} \frac{1}{2} \Gamma_{1} / \Gamma_{2} \pi_{1}(X, *)\right) \otimes \mathbb{R}$. Then:

(i) If $q=0,1, \operatorname{dim} \Gamma_{2} / \Gamma_{3} \pi_{1}(X, *) \otimes \mathbb{R}=0$.

(ii) If $q \geq 2, \operatorname{dim} \Gamma_{2} / \Gamma_{3} \pi_{1}(X, *) \otimes \mathbb{R} \leq 2 q^{2}-7 q+7$.

Proof. We have seen in Cor. 2.10 that $\operatorname{dim} \Gamma_{2} / \Gamma_{3} \pi_{1}(X, *) \otimes \mathbb{R}=\operatorname{dim} \bigwedge^{2} H^{1}(X)-$ $\operatorname{dim} \operatorname{Im}\left(\cup: \bigwedge^{2} H^{1}(X) \rightarrow H^{2}(X)\right)=\frac{2 q(2 q-1)}{2}-\operatorname{dim} \operatorname{Im} \cup$.

Thus if $q=0, \operatorname{dim} \Gamma_{2} / \Gamma_{3} \pi_{1}(X, *) \otimes \mathbb{R}=0$.

If $q=1, \operatorname{dim} \operatorname{Im} U \leq 1$, so $\operatorname{dim} \Gamma_{2} / \Gamma_{3} \pi_{1}(X, *) \otimes \mathbb{R} \leq 1$. Let $a, b$ be a basis of $\Gamma_{1} / \Gamma_{2} \pi_{1}(X, *) \otimes \mathbb{R}$. The equality $\operatorname{dim} \Gamma_{2} / \Gamma_{3} \pi_{1}(X, *) \otimes \mathbb{R}=1$ would imply that $(a, b) \neq 0$ in $\mathcal{L}_{2} \pi_{1}(X, *)$ by Prop. 1.2 (iv). Therefore there would be an isomorphism $\mathcal{L}_{2} F_{2} \stackrel{\cong}{\longrightarrow} \mathcal{L}_{2} \pi_{1}(X, *)$ sending the generators $X_{1}, X_{2}$ of $\mathcal{L}_{2} F_{2}$ to $a, b$ respectively. By Thm. 3.3 this would mean that $\pi_{1}(X, *)$ is not Kähler, leading to a contradiction. Hence our statement follows.

For $q \geq 2$, we break $H^{1}(X)$ into its Hodge components. By Cor. $5.4 \operatorname{dim}(\mathrm{Im}$ $\left.\bigwedge^{2} H^{1,0}(X) \rightarrow H^{2,0}(X)\right) \geq 2 q-3$. The same holds by conjugation for $\bigwedge^{2} H^{0,1}(X) \rightarrow$ $H^{0,2}(X)$. Prop. 5.5 gives the inequality $\operatorname{dim}\left(H^{1,0}(X) \otimes H^{0,1}(X) \rightarrow H^{1,1}(X)\right) \geq$ $2 q-1$ and our statement follows from the addition of bounds.

Prop. 5.6 roughly means that nonfibered Kähler groups need many defining relations. M. Green and R. Lazarsfeld give a bound ([GL],Thm. 5.4), establishing that given $X$ nonfibered compact Kähler in the sense of Def. 5.1, that is admitting no pencil of genus $g \geq 2$, and a presentation of its fundamental group $\pi_{1}(X)=$ $\left\langle x_{1}, \ldots, x_{n} ; r_{1}, \ldots, r_{s}\right\rangle$, then

$$
s \geq n-3
$$

Prop. 5.6 above allows us to establish a more accurate bound:

Corollary 5.7. Let $G=\left\langle x_{1}, \ldots, x_{n} ; r_{1}, \ldots, r_{s}\right\rangle$ be a finite group presentation. If $G=\pi_{1}(X)$, with $X$ nonfibered compact Kähler, and writing $q=\frac{1}{2} b_{1}(X)$, the total number of relations must satisfy

(i) If $q=0, s \geq n$.

(ii) If $q=1, s \geq n-1$.

(iii) If $q \geq 2, s \geq n+4 q-7$.

Proof. The group presentation $G=\left\langle x_{1}, \ldots, x_{n} ; r_{1}, \ldots, r_{s}\right\rangle$ induces an exact sequence $1 \rightarrow N \rightarrow F \rightarrow G \rightarrow 1$ described in (1.3). Let $d_{0}: \oplus \mathbb{R} r_{i} \rightarrow J_{F} / J_{F}^{2} \cong$ $\Gamma_{1} / \Gamma_{2} F \otimes \mathbb{R}$ be the map defined in Prop. 1.7. We may suppose the relations $r_{1}, \ldots, r_{s}$ ordered so that the images of $r_{1}, \ldots, r_{k}$, with $k \leq s$ form a basis of $\operatorname{Im} d_{0} \cong N / N \cap \Gamma_{2} F \otimes \mathbb{R} \hookrightarrow \Gamma_{1} / \Gamma_{2} F \otimes \mathbb{R}$.

By Prop. 1.7 and Cor. $1.8(\mathrm{i}), \operatorname{dim} \Gamma_{1} / \Gamma_{2} G \otimes \mathbb{R}=\operatorname{dim} \Gamma_{1} / \Gamma_{2} F \otimes \mathbb{R}-\operatorname{dim} N / N \cap$ $\Gamma_{2} F \otimes \mathbb{R}$, so there is an equality

$$
n=k+2 q
$$


Let us remark also that dim ker $d_{0}=s-k$.

Thus if $q=0$ we have $n=k \leq s$ as was wanted.

If $q=1$, by Cor. 1.11 and Prop. 5.6 (i) we have that

$$
\begin{aligned}
0 & =\operatorname{dim} \Gamma_{2} / \Gamma_{3} G \otimes \mathbb{R}=\left(\begin{array}{l}
2 \\
2
\end{array}\right)-(s-k)+\operatorname{dim} \operatorname{ker} d_{1} \\
& =1-s+k+\operatorname{dim} \operatorname{ker} d_{1} \geq 1-s+k
\end{aligned}
$$

As $n=k+2$ in this case, this yields the sought bound.

If $q \geq 2$, again by Cor. 1.11 and Prop. 5.6,

$$
\operatorname{dim} \Gamma_{2} / \Gamma_{3} G \otimes \mathbb{R}=\left(\begin{array}{c}
2 q \\
2
\end{array}\right)-(s-k)+\operatorname{ker} d_{1} \leq 2 q^{2}-7 q+7
$$

which implies

$$
k+2 q+\operatorname{dim} \operatorname{ker} d_{1} \leq s-4 q+7
$$

and as $n=k+2 q$,

$$
s \geq n+4 q-7
$$

Examples 5.8.

(i) A group $G=\left\langle x_{1}, \ldots, x_{2 q} ; w_{1}, \ldots, w_{s}\right\rangle$ with $w_{1}, \ldots, w_{s} \in \Gamma_{2} F$ can be nonfibered Kähler only if $s \geq 6 q-7$ for $q \geq 2$, and $s \geq 1$ for $q=1$.

(ii) Chain link groups (see [Ro], 3.G) The group $G_{2 q}=\left\langle x_{1}, \ldots, x_{2 q} ;\left(x_{1}, x_{2}\right), \ldots\right.$, $\left.\left(x_{2 q-1}, x_{2 q}\right),\left(x_{2 q}, x_{1}\right)\right\rangle$ is the fundamental group of a link of $2 q$ circumferences forming a circular chain, for $q \geq 2$. This group verifies $k=\operatorname{dim} \Gamma_{1} / \Gamma_{2} F \otimes \mathbb{R}-$ $\operatorname{dim} \Gamma_{1} / \Gamma_{2} G_{2 q} \otimes \mathbb{R}=0$, and $s=2 q<6 q-7$, and therefore $G_{2 q}$ cannot be nonfibered Kähler. Broadly speaking, if a link is not very intertwined, its group is not going to be nonfibered Kähler. The group $G_{4}$ verifies that $\operatorname{dim} \Gamma_{2} / \Gamma_{3} G_{4} \otimes$ $\mathbb{R}=2$, and therefore it cannot be fibered Kähler either, as it cannot map onto $\pi_{1}\left(C_{g}, *\right)$ for any $g \geq 2$. The groups $G_{2 q}$ with $q \geq 3$ do admit onto mappings to $\pi_{1}\left(C_{2}, *\right)$, and the author does not know if they are fibered Kähler.

(iii) The fundamental group of a compact Riemann surface of genus $g \geq 2$ admits a presentation $\left\langle a_{1}, \ldots, a_{g}, b_{1}, \ldots, b_{g} ;\left(a_{1}, b_{1}\right) \ldots\left(a_{g}, b_{g}\right)\right\rangle$. In this presentation $k=0$, and $s=1<6 g-7$. Therefore, it can only be the fundamental group of a fibered Kähler manifold. This is a particular case of Prop. 5.2.

(iv) Let $G=\left\langle x_{1}, \ldots, x_{5} ; x_{1}^{2} x_{2}^{-2} x_{4}^{2},\left(x_{1}, x_{2}\right),\left(x_{2}, x_{3}\right),\left(x_{3}, x_{4}\right),\left(x_{4}, x_{5}\right)\right\rangle$. In this case $n=5, k=1, q=2$ as $\operatorname{Im} d_{0}=\left\langle 2 \bar{x}_{1}-2 \bar{x}_{2}+2 \bar{x}_{4}\right\rangle$, and $s=5<n+4 q-7=6$. Therefore $G$ cannot be nonfibered Kähler. The group $G$ cannot either map onto $\pi_{1}\left(C_{g}\right)$, with $C_{g}$ a smooth projective curve of genus $g \geq 2$ because $\operatorname{dim} \Gamma_{2} / \Gamma_{3} G \otimes$ $\mathbb{R}=2, \operatorname{dim} \Gamma_{2} / \Gamma_{3} \pi_{1}\left(C_{g}\right) \otimes \mathbb{R}=\frac{2 g(2 g-1)}{2}-1 \geq 5$, so we reach the conclusion that $G$ cannot be Kähler.

\section{Bibliography}


[Be] A. Beauville, Surfaces algébriques complexes ( $3^{m e}$ Ed.), Astérisque 54, S.M.F. 1978.

[Be2] A. Beauville, Appendix to [Ca].

[BG] A. Bousfield, V. Gugenheim, On PL De Rham theory and rational homotopy type, Mem. Amer. Math. Soc. 179, A.M.S. 1976.

[BPV] W. Barth, C. Peters, A. Van de Ven, Compact complex surfaces, Ergeb. Math. Grenzgeb (3) 4, Springer-Verlag 1984.

[C] F. Campana, Remarques sur les groupes de Kähler nilpotents, C. R. Acad. Sci. Paris 317 (1993) 777-780; to appear in Ann. Sci. École Norm. Sup.

[Ca] F. Catanese, Moduli and classification of irregular Kaehler manifolds (and algebraic varieties) with Albanese general type fibrations, Invent. Math. 104 (1991) 263-289.

[Ch] K.T. Chen, Iterated integrals of differential forms and loop space cohomology, Ann. of Math. 97 (1973) 217-246.

[DGMS] P. Deligne, P. Griffiths, J. Morgan, D. Sullivan, Real Homotopy Theory of Kähler Manifolds, Invent. Math. 29 (1975) 245-274.

[GL] M. Green, R. Lazarsfeld, Higher obstructions to deforming cohomology groups of line bundles, J. Amer. Math. Soc. 4 (1991) 87-103.

[G] M. Gromov, Sur le groupe fondamental d'une variété Kählérienne, C. R. Acad. Sci. Paris 308 (1989) 67-70.

[GM] P. Griffiths, J. Morgan, Rational Homotopy Theory and Differential Forms, Progr. Math. 16, Birkhäuser 1981.

[HMR] P. Hilton, G. Mislin, J. Roitberg, Localization of Nilpotent Groups and Spaces, Math. Studies 15, North Holland 1975.

[JR] F. Johnson, E. Rees, On the fundamental group of a complex algebraic manifold, Bull. London Math. Soc. 19 (1987) 463-466.

[JR2] F. Johnson, E. Rees, The fundamental groups of algebraic varieties, in S. Jackowsky et altri (Eds.) Algebraic Topology Poznan 1989, Lecture Notes in Math. 1474, Springer-Verlag 1991, 75-82.

[Ko] T. Kohno, Série de Poincaré-Koszul associée aux groupes de tresses pures, Invent. Math. 82 (1985) 57-75.

[L] M. Lazard, Sur les groupes nilpotents et les anneaux de Lie, Ann. scient. Éc. Norm. Sup. 71 (1954) 101-190.

[M1] J. Morgan, The algebraic topology of smooth algebraic varieties, Inst. Hautes Études Sci. Publ. Math. 48 (1978) 137-204.

[M2] J. Morgan, Hodge theory for the algebraic topology of smooth algebraic varieties, Proc. Sympos. Pure Math. 32 AMS 1978, 119-128.

[Q] D. Quillen, Rational Homotopy Theory, Ann. of Math. (2) 90 (1969) 205-295.

[Q2] D. Quillen, On the Associated Graded Ring of a Group Ring, J. Algebra 10 (1968) 411-418.

[RV] R. Remmert, A. Van de Ven, Zur Funktionentheorie homogener komplexer Mannigfaltigkeiten, Topology 2 (1963) 137-157.

[Ro] D. Rolfsen, Knots and Links, Math. Lecture Ser. 7, Publish or Perish 1990. 
[S] J.-P. Serre, Lie algebras and Lie groups, W.A. Benjamin 1965.

[Siu] Y.T. Siu, Strong Rigidity for Kähler Manifolds and the Construction of Bounded Holomorphic Functions, in R. Howe (Ed.), Discrete Groups in Geometry and Analysis, Progr. Math. 67 Birkhäuser 1987, 124-151.

[St] J.R. Stallings, Quotients of the powers of the augmentation ideals in a group ring, in L.P. Neuwirth, Knots, Groups and 3-Manifolds, Ann. of Math. Stud. 84, Princeton University Press 1975, 101-118.

[W0] R.O. Wells, Comparison of De Rham and Dolbeault cohomology for proper surjective mappings, Pacific J. Math. 53 (1974) 281-300.

[W] R.O. Wells, Differential Analysis on Complex Manifolds, Graduate Texts in Math. 65, Springer-Verlag 1980. 